\title{
Application of Asynchronous Channels Method to the W-CDMA Systems
}

\author{
Etsushi Nameda $^{*}, 1,2$, Hideaki Terai ${ }^{1,2}$, Minghui Kao ${ }^{1,2}$ and Ken Umeno ${ }^{1,2,3}$ \\ ${ }^{1}$ ChaosWare, Inc., Japan \\ ${ }^{2}$ Next Generation Mobile Communications Laboratory, RIKEN, Japan \\ ${ }^{3}$ National Institute of Information and Communications Technology, Japan
}

\begin{abstract}
We evaluate new interference suppression method that utilizes the effect of asynchronous accesses in wireless telecommunication. The method is called as asynchronous channels (ACL), which can be applied to one of the present third generation standards, wideband code division multiple access (W-CDMA) systems. The simulation result shows that the system capacity gains up by $145 \%$ in band-unlimited rectangular pulse situations. On the other hand, in practical bandlimited root raised cosine (RRC) pulse situation, ACL applied system performances are inferior to the conventional synchronous channel systems. Because the another band-limited Gaussian pulse simulation shows better interference than synchronous channels, whether the asynchronous channel can have an effect on interference suppression is highly depending on the choice and the design of pulse shaping filters.
\end{abstract}

Keywords: Asynchronous CDMA, asynchronous channels, multiple access interference, pulse shaping filter.

\section{INTRODUCTION}

Wideband code division multiple access (W-CDMA), also known as universal mobile telecommunications system (UMTS), is one of third generation wireless mobile communication standards, subscribed over 2 billion today and expected 3 billion by 2010 [1]. The significant feature of $\mathrm{W}$ CDMA is the tolerance of asynchronous multiple access in the uplink, the connection from user equipment (UE) transmitters to the Base Station (BS) receiver.

One reason for the asynchronous uplink support in WCDMA is that it was easier to realize continuous system deployment, from outdoors to indoors, with no requirement of any external timing source such as global positioning system [2]. The another reason, relatively not so widely known, is that the asynchronous access suppresses interference noise between multiple mobile stations, revealed by the pioneering analysis of direct sequence CDMA systems by Pursley in 1970's [3].

Pursley's analysis shows that asynchronous access CDMA systems can reduce multiple access interference (MAI) to 2/3 compared with synchronous ones. Though the estimation of interference suppression effects is affected by pulse shapes [4], we remark that the asynchronous is important to design highly efficient mobile communication systems. Our proposal, asynchronous channels (ACL), is a method that aims to utilize such interference suppression effect of the asynchronous access by staggering multiple channels of a user [5].

*Address correspondence to this author at the ChaosWare, Inc.; NICT Collaborative Research Center 2F, Nukui-kitamachi 4-2-1, Koganei, Tokyo 184-8795, Japan; Tel: +81-42-359-6299; Fax: +81-42-359-6339;

E-mail: nameda@riken.jp
In this paper, we report simulation results of ACL applied to W-CDMA systems. In band unlimited situations, ACL demonstrates highly interference suppression effects as expected, decreasing bit error rates (BER) and increasing the system capacity. On the other hand, in band limited situations, the effect of ACL depends on pulse shapes.

The organization of the paper is as follows. In the following section, we introduce the W-CDMA systems and our proposal method, ACL. Next, we discuss simulation results of ACL applied W-CDMA systems on three types of pulse shaping, that is, band-unlimited rectangular pulses, bandlimited root raised cosine pulses and Gaussian pulses. Finally, we draw some concluding remarks and complete the paper.

\section{PRELIMINARIES \\ W-CDMA}

W-CDMA is based on DS-CDMA systems where transmitted symbols are scrambled and spread by pseudo-noise codes. Fig. (1) shows a simplified block diagram of the WCDMA uplink. W-CDMA utilizes two types of code spreading. The first is the channelization operation, which transforms every data symbol into a number of chips, thus increasing the bandwidth of the signal. The ratio of data symbol duration $T_{b .}$. per chip duration $T_{c}$ is called as the spreading factor (SF). The second operation is the scrambling operation, which spread signal broadly to the bandwidth by pseudo-random complex codes denoted as $C_{\text {long }}$ in Fig. (1).

The code used in channelization operation is called as Orthogonal Variable Spreading Factor (OVSF) code. The OVSF code $\mathrm{C}_{\mathrm{ch}, \mathrm{SF}, k}$ is defined by recursively substitutions into the Walsh function such as

$$
C_{\mathrm{ch}, 1,0}=1 \text {, }
$$




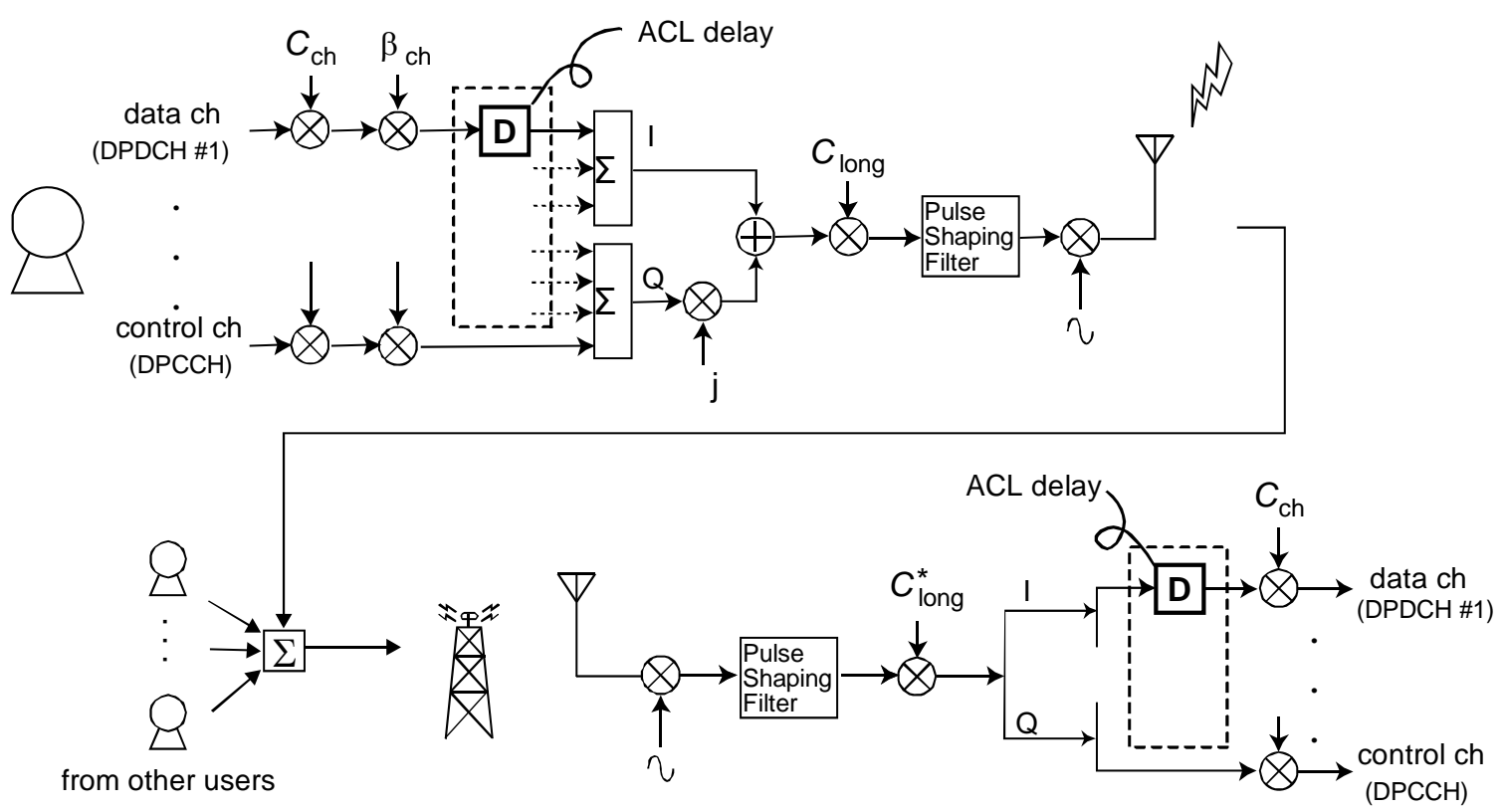

Fig. (1). A simplified block diagram of a user equipment transmitter (upper) and a base station receiver (lower) in the W-CDMA reverse link. ACL delay blocks are also inserted for our proposed extension.

$\left[\begin{array}{l}C_{\mathrm{ch}, 2,0} \\ C_{\mathrm{ch}, 2,1}\end{array}\right]=\left[\begin{array}{ll}C_{\mathrm{ch}, 1,0} & C_{\mathrm{ch}, 1,0} \\ C_{\mathrm{ch}, 1,0} & -C_{\mathrm{ch}, 1,0}\end{array}\right]=\left[\begin{array}{cc}1 & 1 \\ 1 & -1\end{array}\right]$,

$\left[\begin{array}{c}C_{\mathrm{ch}, 2^{(n+1)}, 0} \\ C_{\mathrm{ch}, 2^{(n+1)}, 1} \\ \vdots \\ C_{\mathrm{ch}, 2^{(n+1)}, 2^{(n+1)}-2} \\ C_{\mathrm{ch}, 2^{(n+1)}, 2^{(n+1)}-1}\end{array}\right]=\left[\begin{array}{cc}C_{\mathrm{ch}, 2^{n}, 0} & C_{\mathrm{ch}, 2^{n}, 0} \\ C_{\mathrm{ch}, 2^{n}, 0} & -C_{\mathrm{ch}, 2^{n}, 0} \\ \vdots & \vdots \\ C_{\mathrm{ch}, 2^{n}, 2^{n-1}} & C_{\mathrm{ch}, 2^{n}, 2^{n-1}} \\ C_{\mathrm{ch}, 2^{n}, 2^{n-1}} & -C_{\mathrm{ch}, 2^{n}, 2^{n-1}}\end{array}\right]$,

where $k$ is the code number in $0 \leq \mathrm{k} \leq \mathrm{SF}-1[6]$.

The OVSF codes preserve their orthogonality even in different lengths as far as avoiding their parents and children of recursive generations. Hence, the OVSF codes realize multiple physical channels of various bitrates $[7,8]$.

We often refer the particular code number $k$ of codes that has simple characteristics listed as follows.

- If $k=0$ then all symbols are value 1 .

- If $k=\mathrm{SF} / 2$ then symbol signs alternate every chips.

- $\quad$ If $k=\mathrm{SF} / 4$ then symbol signs alternate every 2 chips.

In W-CDMA systems, there are two kinds of major physical channels, namely, a control channel and data channels. The dedicated physical control channel (DPCCH) is multiplied by the code $C_{\text {ch, } 256,0}$ that every symbol repeats sequential value 1 such as $\{+1+1+1+1+1 \ldots\}$. The dedicated physical data channels (DPDCHs) are typically used only one channel, DPDCH\#1, multiplied by the code number $k=\mathrm{SF} / 4$ that symbols alternate signs every 2 chips such as $\{+1+1-1-1+1+1-1-1 \ldots\}$.

\section{Asynchronous Channels}

ACL is a method to stagger a control channel and data channels on purpose. As illustrated in Fig. (1), we apply ACL to W-CDMA system by inserting delay blocks to DPDCHs. The delaying duration is determined by the parameter, $d$, where $0 \leq d<1$. For example, when employing W-CDMA standard chiprate $R_{\mathrm{c}}=3.84 \mathrm{Mcps}$, the delaying duration is set to $0 \leq d T_{\text {c. }}<0.26 \mu \mathrm{sec}$. As illustrated in Fig. (2), the timings of multiplying channelization code and scrambling codes are off to the side, consequently output sequences are chopped narrower than the standard chip duration $T_{\text {c. }}$. Meanwhile, the DPCCH synchronizes with the scrambling code, so it remains as is.

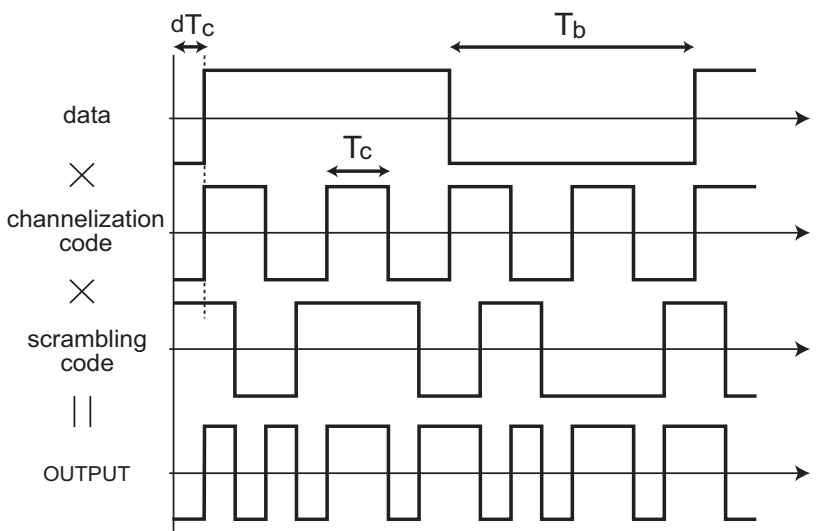

Fig. (2). Time chart of spreading and scrambling with ACL delaying. 


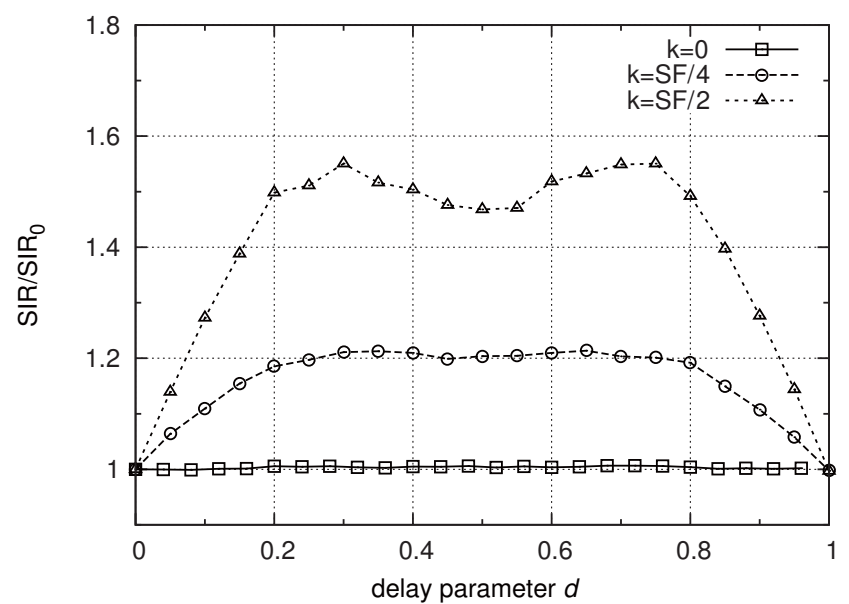

Fig. (3). Comparison of SIR in rectangular pulse for DPDCH\#1 OVSF codes number $k=0, \mathrm{SF} / 4$ and $\mathrm{SF} / 2$ with $\mathrm{Ue}=24$ users and spreading factor $\mathrm{SF}=64$. Each SIR is normalized by the conventional non-delaying $d=0$ case $\left(\mathrm{SIR}_{0}\right)$.

\section{RECTANGULAR PULSE}

First, we begin by the case of the rectangular pulse, without pulse shaping filter. In the rectangular pulse case, as illustrated in Fig. (2), the system output pulses are chopped into two length of pulses, $d T_{\mathfrak{c}}$ and (1-d) $T_{\mathfrak{c}}$, along with OVSF code sign changes. How often pulses are chopped depends on the DPDCH\#1 OVSF code. For example, the code number $k=\mathrm{SF} / 2$ chops every pulses and the code number $k=$ $\mathrm{SF} / 4$ chops one in twice, while the code number $k=0$ never chops.

\section{OVSF Code Dependencies}

In the simulations, we assume all users adopt a common fixed duration for asynchronous channel delay and that generic noises or fading are not concerned because we only focus on multiple access interference.

Fig. (3) shows the simulation result of the ACL applied WCDMA systems signal to interference ratio (SIR) that are normalized by the standard synchronous $d=0$ case of $\mathrm{SIR}_{0}$. and that are plotted along with delaying parameter $d$. The simulated number of user equipments is 24 and the spreading factor is fixed to 64 . When applying the code $C_{\mathrm{ch}, 64,0}$, SIR remains 1 along with delay $d$. This is because the code number $k=0$ does nothing in rectangular pulse situation, therefore, having no effect on ACL. When applying the code $C_{\text {ch, } 64,16}$, SIR increases along the asynchronous channel delay, reaching the maximum gain at the half of chip duration. When applying the code $C_{\text {ch,64,32, }}$, SIR increases larger than the code $C_{\text {ch,64,16 }}$ case, reaching the maximum at both around 0.3 and 0.7 chip duration.

Fig. (4) show bit error rate (BER) against numbers of user equipments in $60 \mathrm{Kbps}$. Comparing the number of $\mathrm{Ue}$ that achieve $\mathrm{BER}=10^{-3}$, we can measure the system capacity gains. The left most plotted lines are the standard W-CDMA. In Fig. (4), the maximum capacity is achieved at $k=\mathrm{SF} / 2$ and delay $d=0.3$ where the system gains up by $145 \%$ relative to the standard W-CDMA. Fig. (5) shows the system capacity gains by ACL on various bitrates from $60 \mathrm{Kbps}$ $(\mathrm{SF}=64)$ to $960 \mathrm{Kbps}(\mathrm{SF}=4)$. The combination of the code
$C_{\text {ch,SF,SF/2, }}, 0.3$ (or 0.7 ) chip delay always performs the highest system capacity gains.

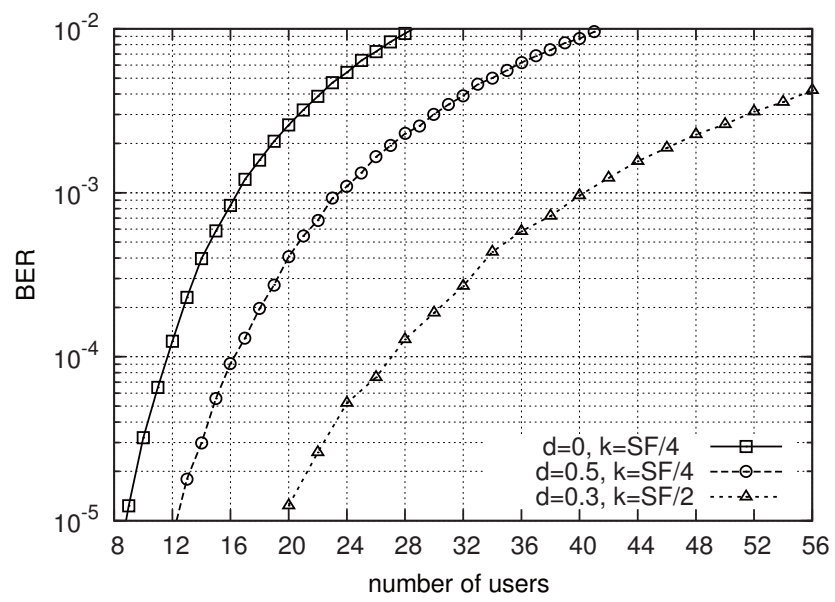

Fig. (4). Comparison of BER in rectangular pulse for the conventional WCDMA system $(d=0, k=\mathrm{SF} / 4)$ and ACL applied systems $(d=0.5, k=\mathrm{SF} / 4)$ and $(d=0.3, k=\mathrm{SF} / 2)$ with spreading factor $\mathrm{SF}$ $=64$.

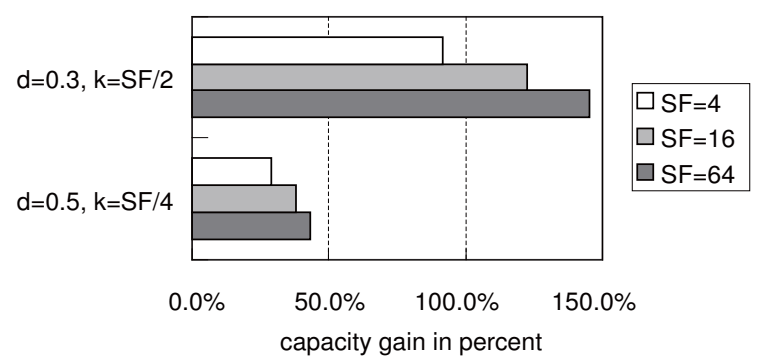

Fig. (5). System capacity gains compared to the conventional WCDMA systems.

\section{Frequency Domain Properties}

The intuitive reason for ACL suppressing multiple access interference is that the system bandwidth is increased by chopped pulses. To confirm this assertion closely, we discuss about frequency domain properties of the ACL applied system on rectangular pulse.

Assuming the code number $k=\mathrm{SF} / 2$, pulses from DPDCH\#1 are divided into reverse signed pairs of length $d T_{\text {c. }}$ and $(1-d) T_{\text {c. }}$ except gaps of data bit duration. The impulse response of the pulse pair can be described as a linear composition of rectangular functions

$h_{\text {rect }}(t)=\operatorname{rect}\left(\frac{t+T_{-} / 2}{T_{+}}\right)-\operatorname{rect}\left(\frac{t-T_{+} / 2}{T_{-}}\right)$

where $\operatorname{rect}(\mathrm{x})=1$ for $-1 / 2 \leq x \leq 1 / 2, T_{+}=d T_{\mathrm{c}}$ and $T_{\mathrm{c}}=$ $(1-d) T_{c}$. Hence, the frequency response $H(f)=\mathrm{F}[h(t)]$ and its power spectrum $|H(f)|^{2}$. are calculated as

$$
\begin{gathered}
H(f)=T_{+} e^{-j \pi f T_{-}} \operatorname{sinc}\left(f T_{+}\right)-T_{-} e^{j \pi f T_{+}} \operatorname{sinc}\left(f T_{-}\right), \\
|H(f)|^{2}=\frac{2 \sin ^{2}\left(\pi f T_{+}\right)+2 \sin ^{2}\left(\pi f T_{-}\right)-\sin ^{2}\left(\pi f T_{c}\right)}{\pi^{2} f^{2}}
\end{gathered}
$$

where $\operatorname{sinc}(x)=\sin (\pi x) / \pi x$. Especially, when taking delay parameter $d=0$ or $d=0.5$, we can simply describe (3) as 


$$
\begin{aligned}
& \left|H_{d=0}(f)\right|^{2}=\frac{1}{\pi^{2} f^{2}} \sin ^{2}\left(\pi f T_{c}\right), \\
& \left|H_{d=0.5}(f)\right|^{2}=\frac{4}{\pi^{2} f^{2}} \sin ^{2}\left(\pi f T_{c} / 2\right) .
\end{aligned}
$$

Fig. (6) shows power spectrums for delay parameter $d=$ $0,0.5$ and 0.3 . Envelope lines for $d=0$ and $d=0.5$ are the coefficients of sine function in (4) and (5), i.e., $\pi^{-2} f^{2}$ and $4 \pi^{-}$ ${ }^{2} f^{2}$, respectively. The power spectrum of the ACL applied system (3) satisfies the inequality

$$
|H(f)|^{2} \leq \frac{4}{\pi^{2} f^{2}}
$$
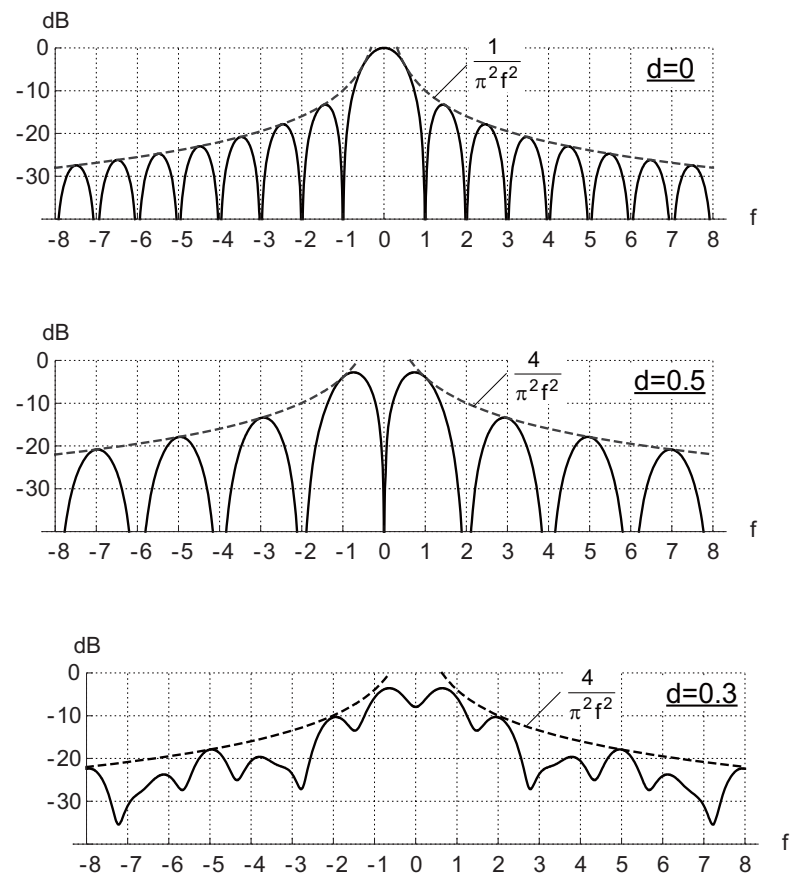

Fig. (6). Power spectrums of the ACL applied rectangular pulse for $d=0, d=0.5$ and $d=0.3$ with the DPDCH\#1 OVSF code number $\mathrm{k}$ $=\mathrm{SF} / 2$.

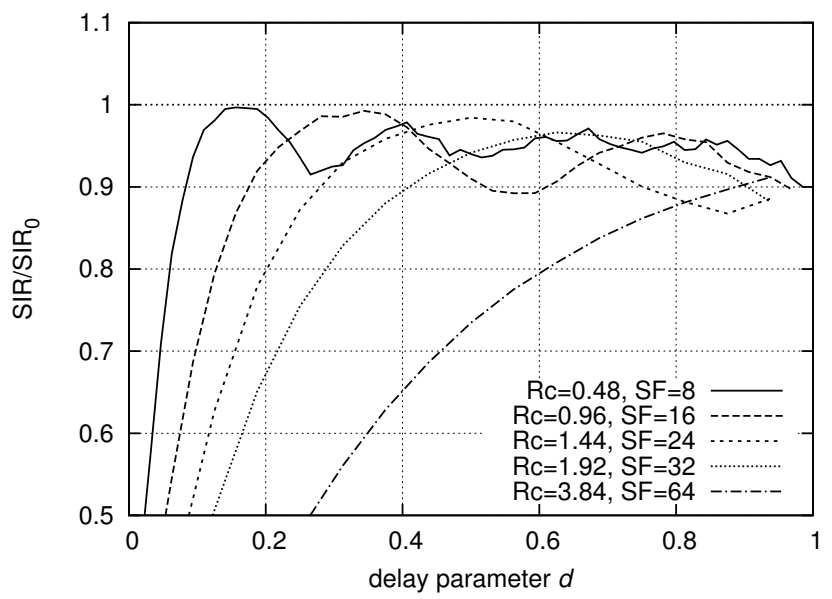

Fig. (7). Comparison of SIR in RRC pulse for various chiprate $R_{c}$ and spreading factor SF combinations with $\mathrm{Ue}=16$ users, the DPDCH\#1 OVSF code number $k=\mathrm{SF} / 2$, roll-off factor $\alpha=0.22$, filter rate $R_{f}=3.84 \mathrm{MHz}$, sampling rate $R_{S}=30.72 \mathrm{MHz}$ and datarate $R_{d}=R_{f} / \mathrm{SF}=60 \mathrm{Kbps}$

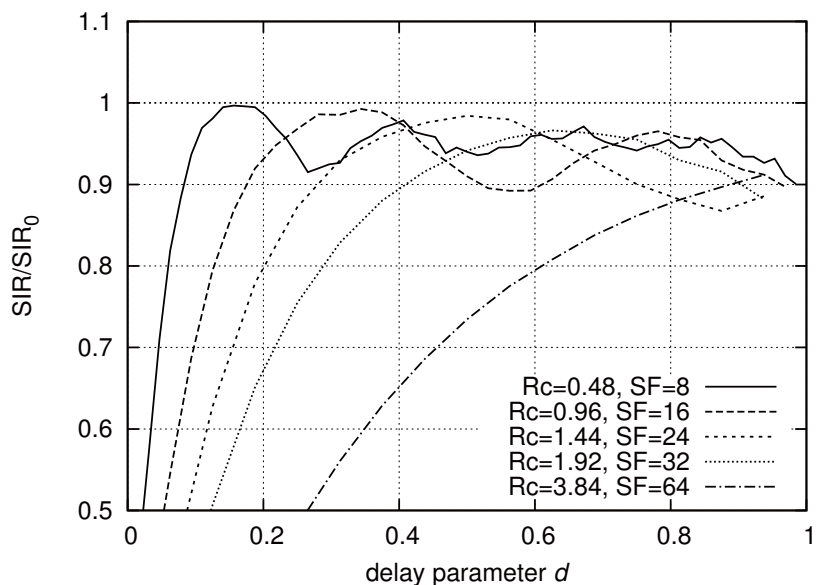

Fig. (8). Comparison of SIR in RRC pulse for various chiprate $R_{c}$ and spreading factor SF combinations with $\mathrm{Ue}=16$ users, the DPDCH\#1 OVSF code number $k=\mathrm{SF} / 2$, roll-off factor $\alpha=0.22$.

as $0 \leq \sin ^{2}(x) \leq 1$, which implies that envelopes for all $d>0$ follows $4 \pi^{-2} f^{2}$. Thus, in terms of power level, the ACL applied system occupies wider bandwidth than the conventional system.

\section{RRC PULSE}

In practice, all radio transmitters are regulated within a given bandwidth. For the case of the present third generation standards, the occupied bandwidth is practically limited in $5 \mathrm{MHz}$. In W-CDMA systems, it employs the root raised cosine (RRC) filter for both a transmitter and a receiver pulse shaping. The impulse response of the RRC pulse is given by

Table 1. The Optimum Delays in Fig. (7)

\begin{tabular}{|c|c|}
\hline $\mathbf{R}_{\mathbf{c}}$ & Delay \\
\hline \hline 0.48 & $0.15625 \approx 1 / 6$ \\
\hline 0.96 & $0.34375 \approx 1 / 3$ \\
\hline 1.44 & $0.5=1 / 2$ \\
\hline 1.92 & $0.625 \approx 2 / 3$ \\
\hline
\end{tabular}

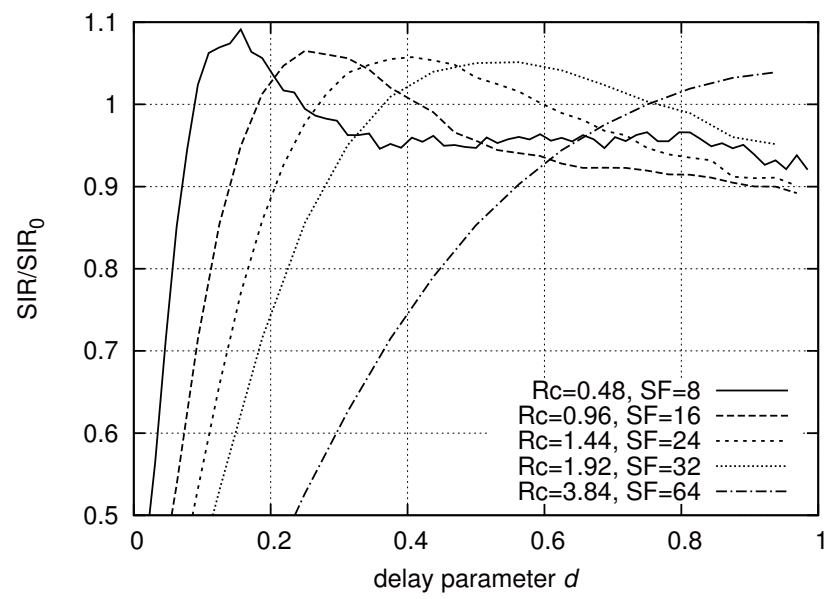

Fig. (9). Comparison of SIR in Gaussian pulse for various chiprate $R_{c}$ and spreading factor $\mathrm{SF}$ combinations with $\mathrm{Ue}=16$ users, filter rate $R_{f}=3.84 \mathrm{MHz}$, roll-off factor $\alpha=0.863$. 


$$
h_{\mathrm{RRC}}(t)=\frac{\sin \left[\pi \frac{(1-\alpha) t}{T_{c}}\right]+\frac{4 \alpha t}{T_{c}} \cos \left[\pi \frac{(1+\alpha) t}{T_{c}}\right]}{\pi \frac{t}{T_{c}}\left[1-\left(\frac{4 \alpha t}{T_{c}}\right)^{2}\right]}
$$

where $\alpha(0 \leq \alpha \leq 1)$ is called as the roll-off factor, the fraction of excess bandwidth [9]. By the RRC filter, the system excess bandwidth is limited to $(1+\alpha) R_{f}$ where $R_{f}$ is the filter standard frequency, and $R_{f}$ is usually equal to the chiprate $R_{c}$. In the W-CDMA specification [10], the roll-off factor $\alpha$ is set to 0.22 , which limits excess bandwidth in $3.84 \mathrm{MHz}$ $\times(1+0.22)=4.6848 \mathrm{MHz}$. The RRC filter is also applied to input signals of the receiver; accordingly, the shaped property of signals is equivalent to the so-called raised cosine filter.

As discussed in the previous section, the ACL method broadens the system bandwidth so we have to regulate the chiprate $R_{c}$. For the comparison, we choose the standard bitrate $R_{b}=60 \mathrm{Kbps}$ settings where $R_{b}=R_{c} / \mathrm{SF}, R_{c}=3.84 \mathrm{MHz}$ and $\mathrm{SF}=64$. The chiprate $R_{c}$ and $\mathrm{SF}$ are both regulated so that the ratio $R_{c}$ per SF stays and the OVSF code for DPDCH\#1 always employs $k=\mathrm{SF} / 2$. Additionally, the filter standard frequency $R_{f}$ is fixed to $3.84 \mathrm{MHz}$, the roll-off factor $\alpha$ is fixed to 0.22 and the filter sampling rate $R_{s}$ is also fixed to $30.72 \mathrm{MHz}$ (x8 over-sample).

Fig. (7) shows simulation results of the ACL applied WCDMA systems SIR, where SIRs are normalized by the each case of delay $d=0$ as Fig. (3), $R_{c}=0.48$ to $3.84 \mathrm{MHz}$ and the number of user equipments are 16. From Fig. (7), we see that the optimum delay $d$ at which the maximum SIR gains changes along with chiprate. These analytical formulas are still unknown, however, the optimum delay and the chiprates might be written as fine rational ratios. Table $\mathbf{1}$ shows the chiprate $R_{c}$ and the optimum delay $d$.

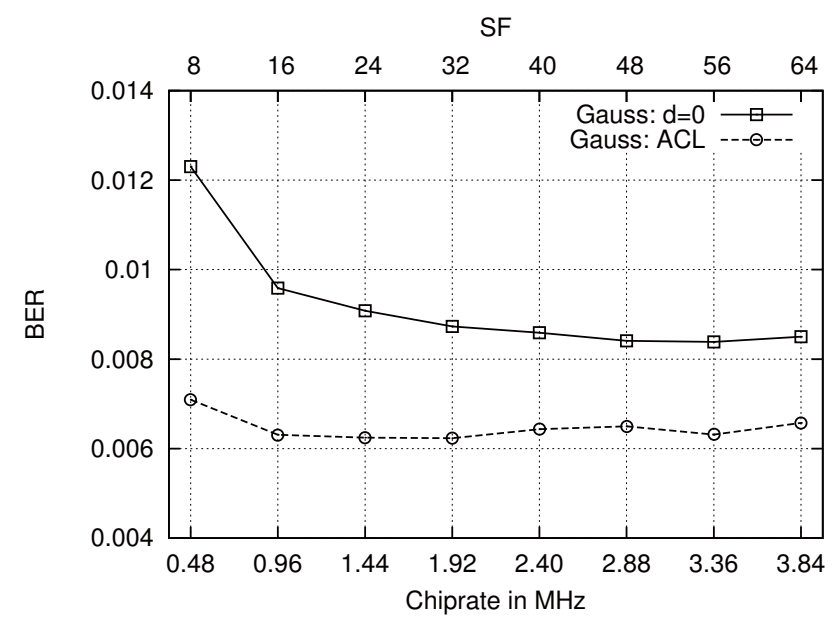

Fig. (10). Comparison of BER in Gaussian pulse for the conventional WCDMA systems $(\mathrm{d}=0)$ and the ACL applied systems employing the optimum delays with $\mathrm{Ue}=16$ users, filter rate $R_{f}=$ $3.84 \mathrm{MHz}$ and roll-off factor $\alpha=0.863$.

Unfortunately, despite from the rectangular pulse case, all SIRs are under 1, that is, the ACL applied RRC pulse systems are inferior in interference performances. Fig. (8) shows the BERs at the optimum delays and also delay $d=0$.
BERs of the ACL applied system reaches the minimum at the half-rate $R_{c}=1.92 \mathrm{MHz}$ of $R_{f}=3.84 \mathrm{MHz}$, which is in contrast to that BERs of synchronous $d=0$ systems monotonically decrease as the chiprate and the spreading factor getting wider.

\section{GAUSSIAN PULSE}

The Gaussian filter is another well-known pulse shaping filter, used in the Global System for Mobile communications (GSM) systems. In this section, apart from the W-CDMA standard regulations, we adopt the Gaussian pulse for ACL applied systems.

The impulse response of the Gaussian pulse is given by

$$
h_{\text {Gauss }}(t)=\sqrt{\frac{\alpha}{T_{c}}} \sqrt[4]{2} \exp \left[-\pi\left(\frac{\alpha t}{T_{c}}\right)^{2}\right]
$$

where $\alpha$ is the roll-off factor. The frequency response of the Gaussian pulse is also the Gaussian function, so it has infinitely ranges in the frequency domain. Nevertheless, the frequency response of the Gaussian pulse decays still faster than that of the rectangular pulse.

For comparing with RRC pulse, we adopt the roll-off factor for Gaussian $\alpha=0.863$ so that an excess band energies becomes the same as a RRC pulse of $\alpha=0.22$. The excess band energy is defined as the integral of the frequency response within $-R_{f} / 2$ and $R_{f} / 2$ in the frequency domain. In this case, the ratio of the excess band energy per the whole band energy is calculated as 0.960 [11].

Fig. (9) shows simulation results of the ACL applied Gaussian pulse systems SIR per $\operatorname{SIR}_{0}$ of delay $d=0$. The parameters, such as the number of users, the filter standard rate $R_{f}$ or the chiprate $R_{c}$ settings etc., are the same as Fig. (7) except the pulse shape and the roll-off factor $\alpha$. It is easy to see that SIR curves in Fig. (9) are slightly differs from the RRC in Fig. (7). The optimum delays for each chiprates are also smaller than RRCs. Table 2 shows the chiprate $R_{c}$ and the optimum delay $d$ in the Gaussian pulse.

Table 2. The Optimum Delays in Fig. (9)

\begin{tabular}{|c|c|}
\hline $\mathbf{R}_{\mathbf{c}}$ & Delay \\
\hline \hline 0.48 & $0.15625 \approx 1 / 6$ \\
\hline 0.96 & $0.25=1 / 4$ \\
\hline 1.44 & $0.40625 \approx 2 / 5$ \\
\hline 1.92 & $0.5625=9 / 16$ \\
\hline
\end{tabular}

In the case of Gaussian pulse, SIR ratios always exceed 1 , so the ACL have an effect on interference suppressions. Fig. (10) shows the BERs at the optimum delays and also delay $d=0$. BERs of the ACL applied system totally stay below delay $d=0$. BERs of the ACL applied system quickly reaches bottom at $R_{c}=0.96 \mathrm{MHz}$ and totally stays below the conventional delay $d=0$ systems. However, the minimum BER of the ACL applied Gaussian pulses 
$\mathrm{BER}_{\text {Gauss }+\mathrm{ACL}}=0.0062$

is still higher than the standard RRC pulse WCDMA systems

$\mathrm{BER}_{\mathrm{RRC}}=0.0040$

in $R_{c}=3.84 \mathrm{MHz}$, and even slightly inferior to the minimum BER of the ACL applied RRC systems

$\mathrm{BER}_{\mathrm{RRC}+\mathrm{ACL}}=0.0058$.

The total BER order of our simulation comes to

$\mathrm{BER}_{\mathrm{RRC}}<\mathrm{BER}_{\mathrm{RRC}+\mathrm{ACL}} \leq \mathrm{BER}_{\mathrm{Gauss}+\mathrm{ACL}}<\mathrm{BER}_{\mathrm{Gauss}}$.

\section{CONCLUSION}

We investigate the performance of the asynchronous channels method, which staggers a timing of multiplying data symbols and scrambling symbols on purpose. This intensive asynchronism comes from known analysis of interference suppression effects of the asynchronous access derived by Pursley. We evaluate numerically the performance of the ACL method applied to W-CDMA systems.

The simulation result of band-unlimited rectangular pulse situation on which the Pursley's analysis based shows that the ACL method can significantly increase the system capacity. Applying the ACL, the system capacity gains up by $145 \%$ with the combination of 0.3 (0.7) chip delays and the OVSF code number $k=\mathrm{SF} / 2$ for a data channel. This is because the asynchronous multiplications of scrambling codes and data chips duplicate pulses, which increases seeming chiprates and widen the occupied bandwidth.

In [12], we obtained an analytical result that asynchronous half delay maximizes SIR in a simple generic DSCDMA model. However, as the W-CDMA model investigated in this paper is more sophisticated system, the reason why the odd delay like 0.3 performs the best interference suppression is still unclear.

We need precise auto- and mutual-correlation analysis on divided chips that has randomness induced by scrambling but also has strong short correlation induced by orthogonal spreading.

Together with band-limited pulse shaping, the ACL applied systems have disadvantages comparing to the conventional W-CDMA systems. With RRC pulse filter, BER of the
ACL applied systems reaches the minimum at the half of filter rate but BER totally stay above the conventional synchronous systems. The RRC pulse is natively designed to free from inter symbol interference of the transmitter itself. The ACL application might break this property, which excess a benefit of multiple access interference suppression in the case of a RRC pulse. On the other hand, with Gaussian pulse filter, BER of the ACL applied system totally stay below the synchronous systems. Gaussian pulse is the pulse that has long monotonic decays and peculiar inter symbol interference. The ACL could reduce this interference.

However, the total BER performance of the ACL applied Gaussian pulse still inferior to RRC pulse.

It will be our future works that analyze SIR function precisely and find better combinations of the ACL and pulse shaping. By this way, if it can halve the chiprate of the system, it will be another solution to the electric power consumption problem of the next generation mobile communication equipments.

\section{REFERENCES}

[1] P. Rysavy, "EDGE, HSPA \& LTE", 3G Americas, White Paper, Sep 2006. [Online] Available from: http://www.3gamericas.org

[2] F. Adachi, M. Sawahashi, and H. Suda, "Wideband DS-CDMA for next-generation mobile communications systems", IEEE Commun. Mag., vol. 36, no. 9, pp. 56-69, Sep 1998.

[3] M. B. Pursley, "Performance evaluation for phase-coded spreadspectrum multiple-access communication-part I: System analysis", IEEE Trans. Commun., vol. 25, pp. 795-799, 1977.

[4] R. Kohno, R. Meidan, and L. B. Mislstein, "Spread spectrum access methods for wireless communication", IEEE Commun. Mag., vol. 33, no. 1, pp. 58-67, Jan 1995.

[5] K. Umeno, Japanese Patent 3650 821, Oct 21, 2004.

[6] Spreading and modulation (FDD) (Release 6), 3GPP TS 25.213, Rev. 6.5.0, Mar 2006.

[7] F. Adachi, M. Sawahashi, and K. Okawa, "Tree-structured generation of orthogonal spreading codes with different lengths for forward link of DS-CDMA mobile radio", IEE Electron. Lett., vol. 33, pp. 27-28, Jan 1997.

[8] K. Okawa, and F. Adachi, "Orthogonal forward link using orthogonal multi-spreading factor codes for coherent DS-CDMA mobile radio", IEICE Trans. Commun., vol. E81-B, no. 4, pp. 777784, Apr 1998.

[9] J. S. Lee, and L. E. Miller, CDMA Systems Engineering Handbook. Boston: Artech House Publishers, 1998.

[10] User Equipment (UE) radio transmission and reception (FDD)(Release 1999), 3GPP TS 25.101, Rev. 3.19.0, Dec. 2006.

[11] Y. Jitsumatsu, M. T. A. Khan, and T. Kohda, "Gaussian chip waveform together with markovian spreading codes improve BER performance in chip-asynchronous CDMA systems", In: Proc. IEEE Globecom '06, San Francisco, CA, Nov 2006, pp. 1-5.

[12] K. Umeno, and R. Takahashi, "Optimal asynchronous channel (ACL) method for multi-carrier communication systems", In: Proc. of $22^{n d}$ IEICE SIP SYMPOSIUM, Sendai, Japan, Nov 2007, pp. 538-541, (in Japanese).

This is an open access article licensed under the terms of the Creative Commons Attribution Non-Commercial License (http://creativecommons.org/licenses/by$\mathrm{nc} / 3.0 /$ ) which permits unrestricted, non-commercial use, distribution and reproduction in any medium, provided the work is properly cited. 